\title{
Research on compound comprehensive energy evaluation and intelligent control optimization
}

\author{
Tiecheng $\mathrm{Li}^{1}$, Siming Zeng ${ }^{1, *}$, Zhengfu Yang ${ }^{2}$, Zhibin $\mathrm{Liu}^{3}$, and Yajing $\mathrm{Liu}^{3}$ \\ ${ }^{1}$ Electric Power Research Institute State Grid Hebei Electric Power Co., Ltd., 050000 Shijiazhuang, \\ China \\ ${ }^{2}$ State Grid Xiong'an Digital Technology Co., Ltd, 05000 Shijiazhuang, China \\ ${ }^{3}$ School of Electronic and Control Engineering, North China Institute of Aerospace Engineering \\ 065000 Langfang, China
}

\begin{abstract}
Under the background of increasing energy demand, integrated energy system is an important way to promote the development of clean energy, support the efficient use of energy and energy saving and emission reduction. This paper firstly establishes a comprehensive energy system evaluation index model composed of three sub-models of economy, environment and energy. On the basis of this model, a comprehensive energy multi-objective optimization mathematical model with the lowest total cost, excellent environmental benefits and high comprehensive energy utilization efficiency is established, and the energy supply and demand allocation optimization method based on improved genetic algorithm is adopted for solution. The simulation proves the effectiveness of the proposed optimization model.
\end{abstract}

\section{Introduction}

With the increasing depletion of traditional energy sources and the growing prominence of environmental pollution, the efficient use of renewable energy has become the key to solving the energy crisis in today's society $[1,2]$. However, optimizing the use of a single energy source is not only inefficient but also costly, so establishing a comprehensive and complementary energy system and giving full play to the complementary advantages of multiple energy sources is a strategic move to ensure energy security and national security [3-5].

The modeling and analysis, operation and scheduling optimization, and comprehensive evaluation of integrated energy have become hot spots for scholars at home and abroad. Zheng et al [6] studied a large-scale integrated energy system composed of distributed district heating and cooling units and wind turbines, and proposed a multi-target group search optimization method to optimize the system's power scheduling. An approximate solution algorithm for the real-time scheduling optimization problem is proposed for an integrated energy system equipped with intermittent renewable energy sources in literature [7]. Hao Ran et al [8] established a two-tier optimization model for integrated energy system to reduce cost and improve energy efficiency, and applied a fuzzy integrated

${ }^{*}$ Corresponding author: $17692149858 @ 139 . c o m$ 
decision method to choose the best one.

This paper first establishes a comprehensive energy system evaluation index model, and on the basis of this model, solves the comprehensive energy optimization model from the perspectives of economy, environmental index and comprehensive energy utilization rate.

\section{Introduction to the complementary coordination program for integrated energy systems}

The evaluation index of comprehensive energy system is an important basis for system planning and design and optimization of regulation and control, and a reasonable evaluation index structure is conducive to the planning and operation of comprehensive energy system.

The comprehensive energy system evaluation index used in this paper consists of three sub-models: economy, environment and energy. Through the settings of the three submodels, the hierarchical combination forms a reasonable criterion for the planning and regulation of the comprehensive energy system.

\section{Multi-objective optimization model for energy supply and demand allocation}

The mathematical model for multi-objective optimization of energy supply and demand allocation takes economic benefits, environmental benefits, etc. as optimization objectives, the supply of different grades of energy from each energy facility as optimization variables, and the balance between supply and demand as constraints.

\subsection{Model multi-objective functions}

Economic objectives are the focus of attention during the planning phase of an integrated energy system, and an acceptable economic input is necessary to support the feasibility planning of an integrated energy system. The economic efficiency objective function can be expressed as:

$$
\begin{gathered}
\min f_{1}(x)=\min \left[\sum_{i=1}^{M}\left(C_{i n v, i}+C_{o p r, i}+C_{f u e l, i}\right)\right] \\
C_{i n v, i}=C_{i n, i} \cdot \frac{R(1+R)^{l}}{(1+R)^{l}-1}=U_{i} P_{i} \cdot \frac{R(1+R)^{l}}{(1+R)^{l}-1} \\
C_{o p r, i}=\epsilon_{i} C_{i n, i} \\
C_{f u e l, i}=\sum_{j=1}^{N}\left(B_{i, j} \sigma_{j}\right) \cdot \sum_{k=1}^{T}\left(\varepsilon_{i, k} t_{i, k} P_{i}\right)
\end{gathered}
$$

where $C_{i n v, i}, C_{o p r, i}, C_{f u e l, i}$ denote the initial investment cost, operation and maintenance cost, and fuel cost of energy equipment of class $i . C_{i n, i}$ is the initial investment cost, $R$ is the discount rate, $l$ is the life cycle, $U$ is the unit price of thermal output, $P$ is the rated load, $\epsilon$ is the operation and maintenance investment factor, $B$ is the amount of fuel consumed, $\sigma$ is the purchased cost, $\varepsilon$ is the average load factor and $t$ is the total run time.

This paper treats the environmental benefits as an objective function independent of the economic benefits. 


$$
\begin{aligned}
\min f_{2}(x) & =\min \left[\sum_{i=1}^{M} \sum_{j=1}^{N}\left(E_{i, j} \cdot T_{i} \cdot \delta_{j}\right)\right] \\
\mathrm{Q} T_{i} & =\sum_{k=1}^{T}\left(\varepsilon_{i, k} t_{i, k} P_{i}\right)
\end{aligned}
$$

where $E_{i, j}$ is the $j$ pollutant emissions per unit load of the equipment of the class $i$ energy unit, $\mathrm{Q} T_{i}$ is the total annual load of the class $i$ energy unit, and $\delta_{j}$ is the equivalent emission factor of the class $j$ pollutant.

\subsection{Model constraints}

In order to make the optimization results meet the many constraints in the actual planning, the range of the optimization variables themselves and the constraint relationship between multiple variables should be considered.

(1) Heat load supply-demand balance constraint

There are two main categories of demand for steam $Q_{\text {steam.demand }}$ and demand for hot water $Q_{\text {water.demand }}$.

$$
\begin{aligned}
& \sum_{i=1}^{M} Q_{\text {steam. } i}=Q_{\text {steam.demand }} \\
& \sum_{i=1}^{M} Q_{\text {water. } i}=Q_{\text {water.demand }}
\end{aligned}
$$

(2) Cold load supply-demand balance constraint

$$
\sum_{i=1}^{M} Q_{c . i}=Q_{c . \text { demand }}
$$

(3) Equipment capacity constraint

Constraints on the upper and lower limits of heating and cooling capacity of various energy unit equipment:

$$
\begin{aligned}
& P_{h . i}^{\min } \leq P_{h . i} \leq P_{h . i}^{\max } \\
& P_{c . i}^{\min } \leq P_{c . i} \leq P_{c . i}^{\max }
\end{aligned}
$$

\subsection{Model solving based on improved genetic algorithms}

This paper improves the basic genetic algorithm for better application in the solution of energy supply and demand configuration optimization problems.

(1) Adaptation function correction based on simulated annealing

$$
f i t(x)_{i m}=\exp \left[-\frac{1}{k^{t} \cdot T_{0} \cdot f(x)}\right]
$$

where $f i t(x)_{i m}$ is the adaptation function, $k$ is the simulated annealing function coefficient, $t$ is the evolutionary algebra of the genetic algorithm, $T_{0}$ is the simulated annealing initial temperature, and $f(x)$ is the objective function value.

(2) Adaptive cross probability and variation probability follows.

The adaptive crossover probability and the probability of variation are expressed as

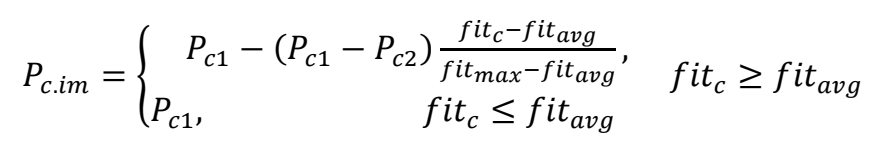




$$
P_{m . i m}=\left\{\begin{array}{c}
P_{m 1}-\left(P_{m 1}-P_{m 2}\right) \frac{f i t_{m}-f i t_{\text {avg }}}{\text { fit }_{\text {max }}-f i t_{\text {avg }}}, \\
f i t_{m} \leq f i t_{\text {avg }}
\end{array} \quad \text { fit }{ }_{m} \geq f i t_{\text {avg }}\right.
$$

\section{Simulation}

In this paper, the integrated energy system planning research and analysis is carried out for a medical port town. Set the simulated annealing-based adaptation function coefficients in the adaptation function correction as follows.

$$
\mathrm{k}=\left\{\begin{array}{c}
1, t \leq \frac{t_{\max }}{2} \\
0.99^{t}, t>\frac{t_{\max }}{2}
\end{array}\right.
$$

Assuming that the population size $M$ is 100 , the maximum number of iterations $t_{\max }$ is 500, and the upper and lower bounds of the adaptive crossover and variation probabilities are $\mathrm{P}_{\mathrm{c} 1}=0.9, \mathrm{P}_{\mathrm{c} 2}=0.6, \mathrm{P}_{\mathrm{m} 1}=0.1, \mathrm{P}_{\mathrm{m} 2}=0.001$.

The energy structure evaluation indicators described in Chapter 2 are used to analyse the utilization rate of recycled energy for the corresponding scenario of the Pareto solution set and the results are shown in Figure 1.

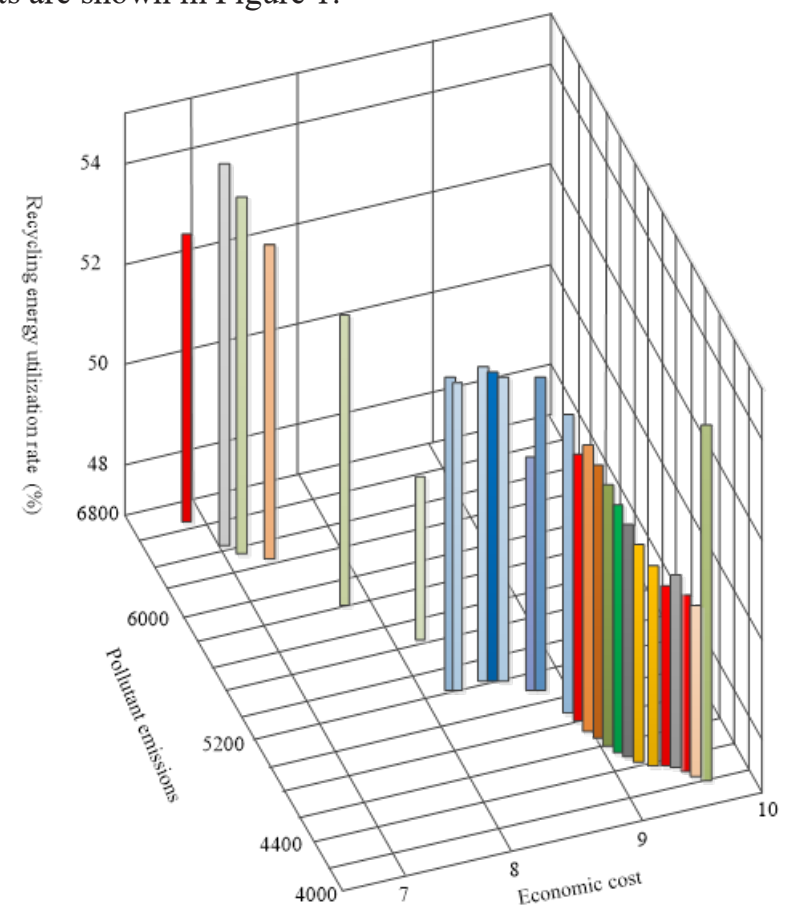

Fig. 1. Recycling energy utilization rate of Pareto solution optimization of energy supply and demand allocation.

\section{Conclusion}

In this paper, the comprehensive energy system evaluation index model including economic, environmental and energy structure elements is studied. The economic index based on the comprehensive cost method is used to evaluate the economic benefit optimization degree 
under the condition of multi energy allocation. The simulation results show that the Pareto solution set of energy supply and demand allocation optimization oriented to economic and environmental objectives is obtained. At the same time, the energy structure evaluation index is used to analyze and understand the recycling energy utilization rate of the set scheme. The results show that, in general, the utilization rate of recycling energy is more than $45 \%$.

Science and Technology Project of State Grid Corporation of China, kjcb-2020-45. Research on key technology of integrated energy system with heat storage device.

\section{References}

1. International Energy Agency, District Energy Systems in China, [EB/OL] https://webstore.iea.org/district-energy-systems-in-china-chinese.

2. Wei X G, Gao Sh B, Zang T L, etc. Social Energy Internet: Concept, Architecture and Prospect [J]. Proceedings of the Chinese Electrical Engineering, 2018, 38 (17): 49694986, 5295.

3. Fang B L. Multi-energy complementation and integration optimization of hybrid renewable energy systems [D]. Changsha: Hunan University, 2017.

4. Peng K, Zhang $\mathrm{C}, \mathrm{Xu} \mathrm{B} \mathrm{Y}$, et al. Current status and prospects of multi-energy collaborative integrated energy system demonstration project [J]. Electric Power Automation Equipment, 2017, 37(6):3-10.

5. Mittal, Saurabh, Ruth, et al. System-of-Systems Approach for Integrated Energy Systems Modeling and Simulation[C]// Conference on Summer Computer Simulation. Society for Computer Simulation International, 2015.

6. Zheng J H, Chen J J, Wu Q H, et al. Multi-objective optimization and decision making for power dispatch of a large-scale integrated energy system with distributed DHCs embedded [J].Applied Energy, 2015, 154:369-379.

7. Firestone R, Stadler M, Marnay C. Integrated Energy System Dispatch Optimization[C]// IEEE International Conference on Industrial Informatics. IEEE, 2006.

8. Hao R, Ai Q, Zhu Y CH, et al. Hierarchical optimal dispatching of regional integrated energy system based on energy hub[J]. Electric Power Automation Equipment, 2017, 37(6):171-178. 